\title{
Computer as a tool in astronomy teaching
}

\author{
By Francis BERTHOMIEU
}

\author{
Lyce Jean Moulin, Place de la Paix, 83300 DRAGUIGNAN, FRANCE
}

As yet, astronomy, the most ancient of all sciences, surprisingly is not included in French secondary science classes. Recent trends in favour of a more attractive and motivating scientific education have taken it up.

Astronomy has, at all times, been arising curiosity, and now provides a privileged field to scientific approach :

- Observation of the vault of heaven and its peculiarities

- Description of its general appearance and of the specific movement of stars and planets

- Measurement of distances, coordinates and angles.

- This will make it possible to define successive models, which will be ever closer to the observed reality.

The obstacle of mathematics must be avoided or bypassed : many devices and demonstration models allow for a simplified and convincing approach. Computers may be valuable tools. My purpose is not to go through the multimedia version of an encyclopaedia but to follow some new trails.

DIGITAL IMAGES are efficient tools for first experiences : observation can be adapted to a specific public and digital images can guide pupils through observation. They facilitate measuring operations : interaction will incite users to creativity and discovery, and numerical models will be exploited much more easily.

The movement of planets is a quite convincing example. I use for that purpose a series of digital images of the sky : each photograph represents the constellation of Taurus, all taken during the 1990-1991 winter. My software allows pupils to recognize the characteristic stars of that region and to locate the moving planet Mars among them.

With a mouse click, the position of Mars is transfered to a map of the sky, that was conceived in order to represent the constellation of Taurus such as it would appear on a photograph. Beside that, the map includes the celestial coordinates, so that the pupils can also perform some double measurements. In addition, the software constructs, point by point, the apparent trajectory of the planet and the pupils become convinced that this movement really takes place near the Ecliptic plane (see Fig. 1).

Another example can be that of the Moon. Many people confuse the explanation of its phases with that of eclipses. Our younger pupils can discover the difference playing with a ball and a projector. A formal explanation involves some difficult 3-D geometrical construction, which can be easily presented by computers. Digital pictures of the Moon were taken in the course of one month, and introduced into other software. The user can easily draw the circular border of the Moon. Then the program allows you to choose the position of the Sun so that the drawing of the Moon looks like the photograph. You obtain also the size of the circle and the value of the angle between the axes Moon-Earth and Moon-Sun. Nothing remains but to find the reason why the size of the Moon apparently changes during the month, and how it moves in relation to its celestial neighbours, the Earth and the Sun! (See Fig. 2, 3.)

MOVING PICTURES are another means for visualizing astronomical concepts. Even though underlying mathematics are sometimes out of the users reach, moving pictures facilitate the understanding of the phenomena. They are a solution particularly well- adapted to geometrical models.

Unfortunately Figure 4 shows a static copy of the computer screen. The pupil, indeed, can observe and analyse the moving picture. He notes that six moving points determine two triangles. These triangles get distorted but remain identical one to another. As one of their vertices does not move, the program draws the trajectory of the others.

It is a great pleasure to detect the pupils pleasure when they suddenly discover that these 


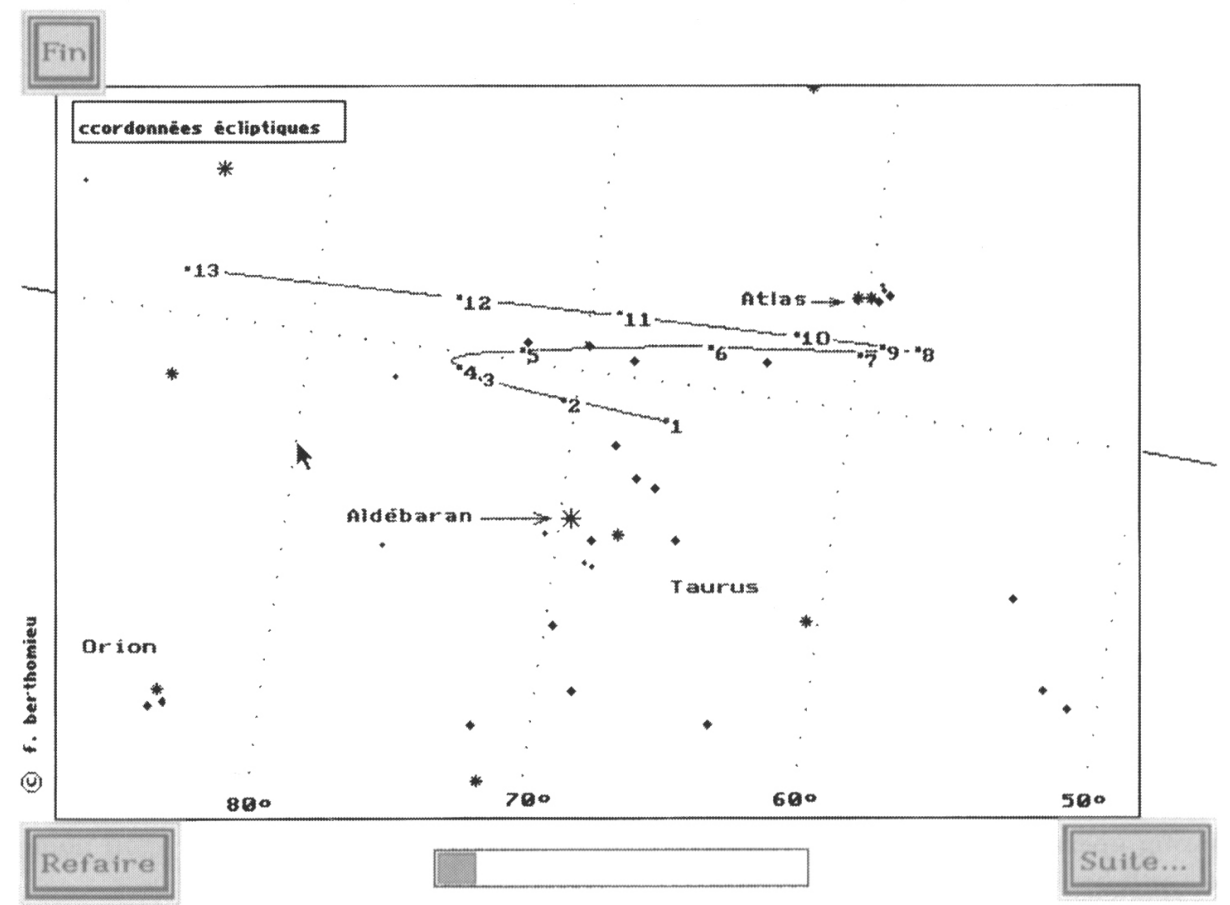

Figure 1. The track of Mars in the constellation of Taurus.

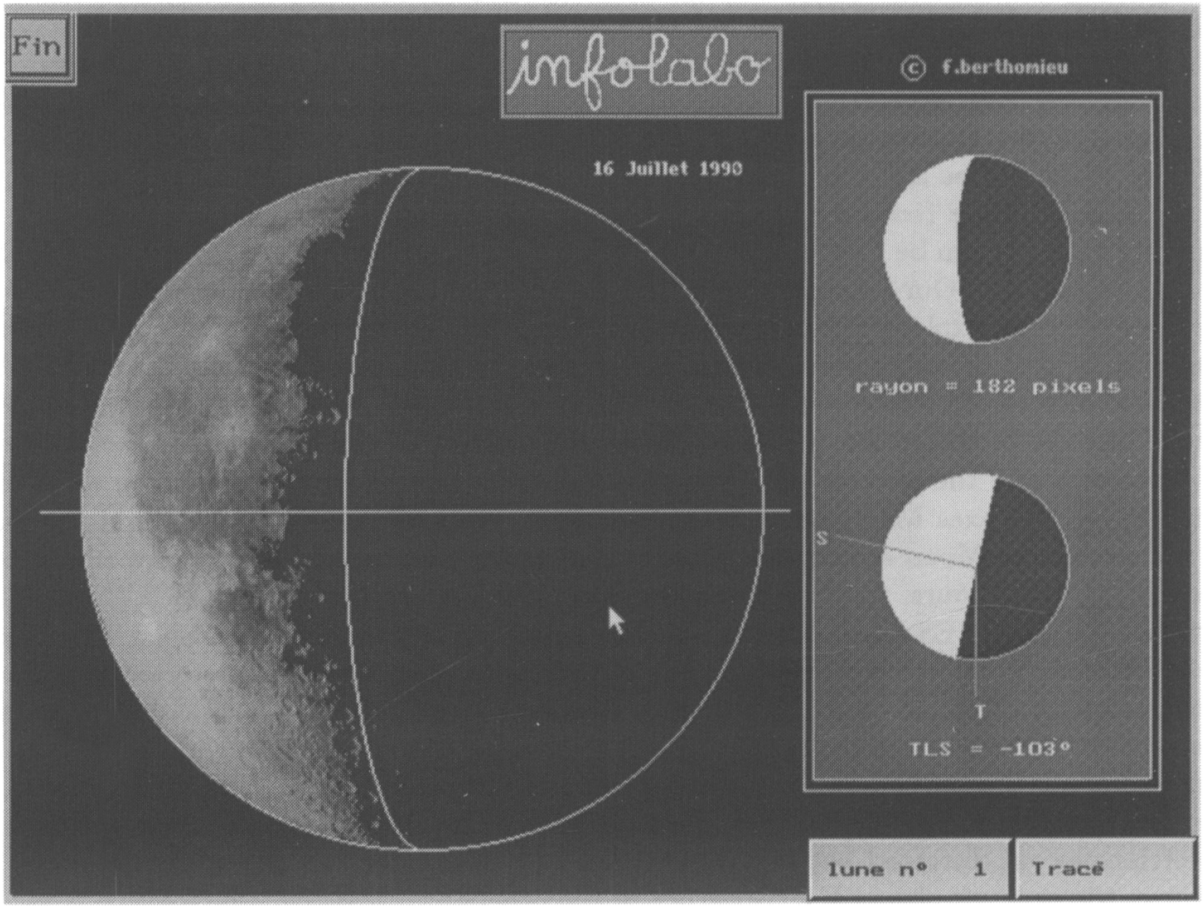

Figure 2. Phases of the Moon. 


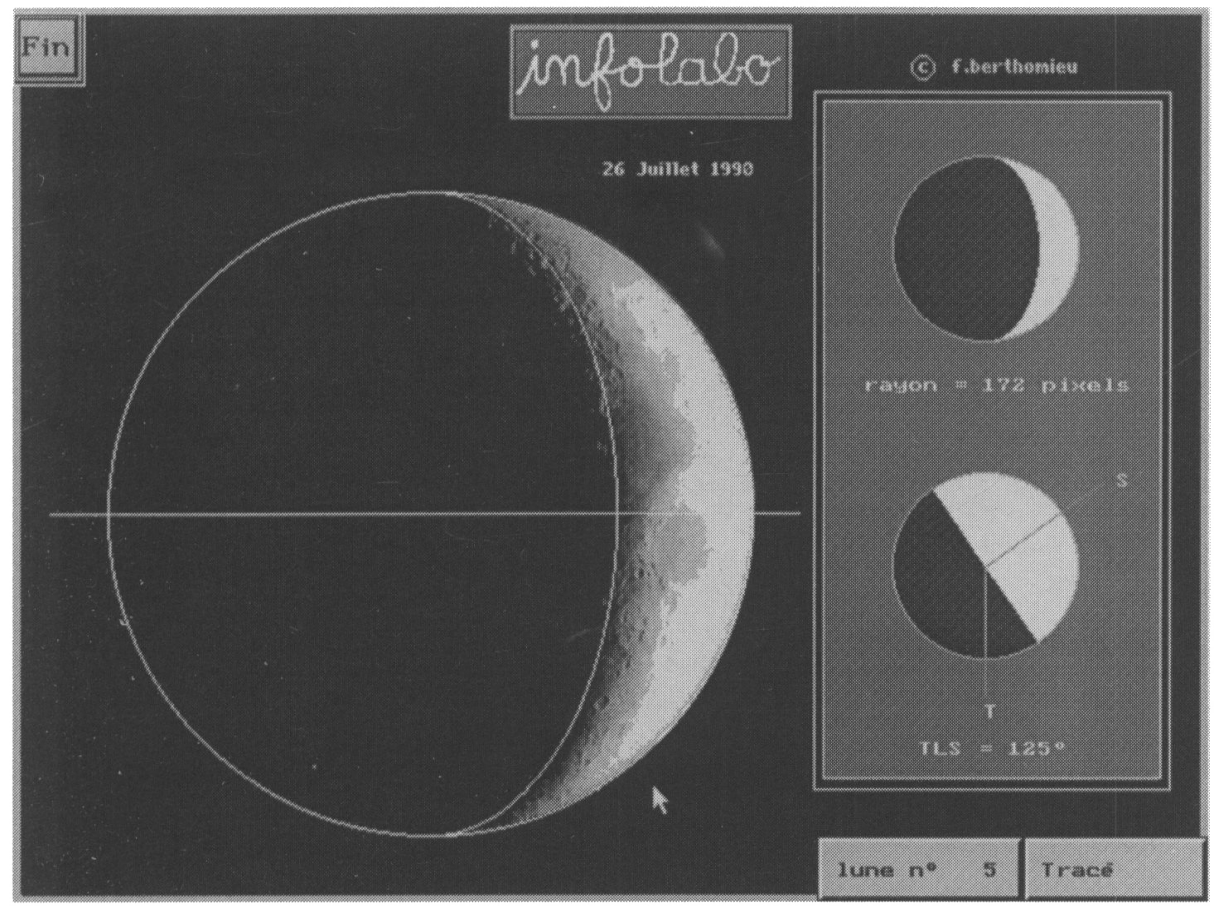

Figure 3. Phases of the Moon.

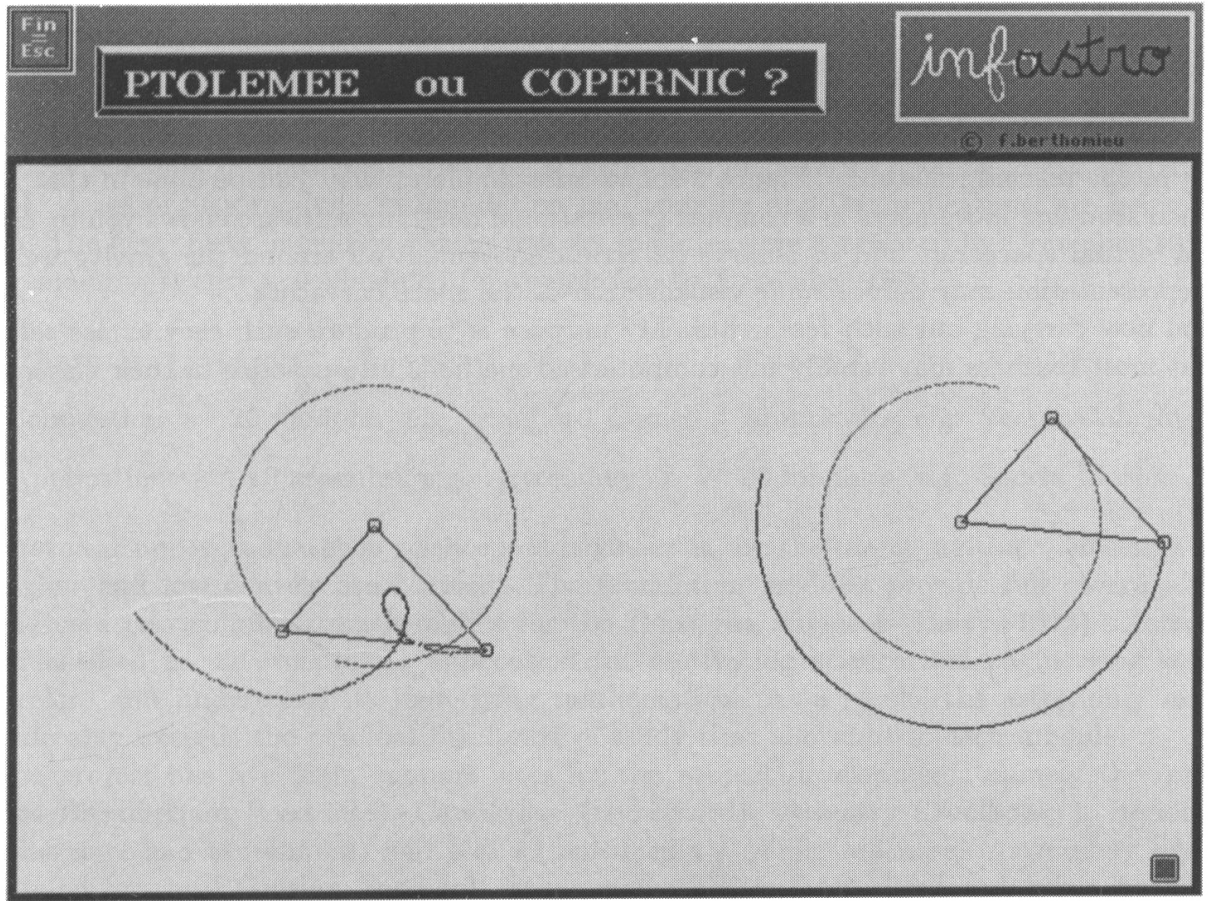

Figure 4. Geocentre and Heliocentre Motion. 


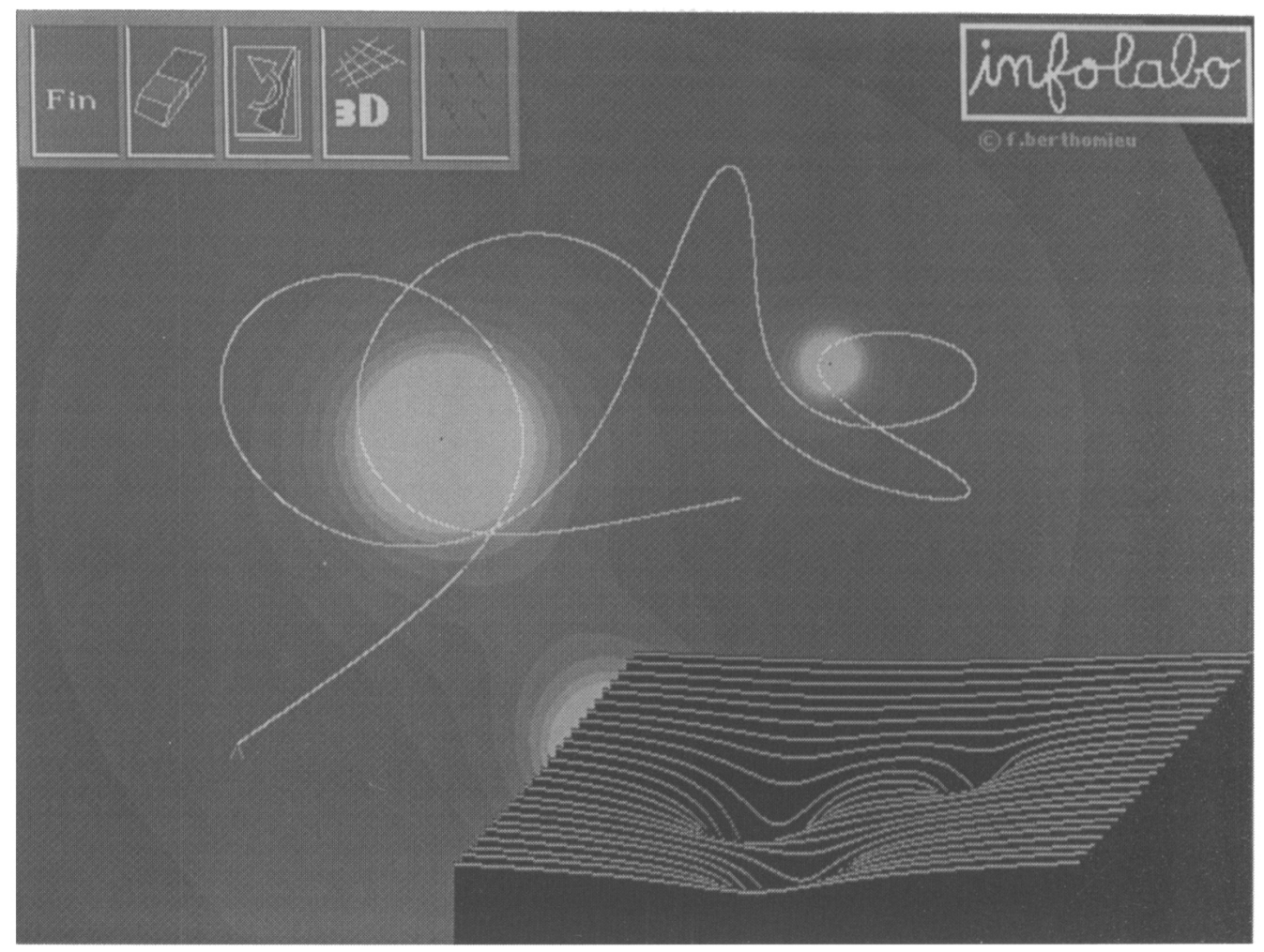

FIGURE 5. Illustration of Orbital Motion.

points on the screen can be identified with the Sun, the Earth and Mars : it is the exact moment when they understand for themselves the difficult problem of Solar System models.

While scientific research has been using it for decades, numerical simulation is coming (too shyly) in our teaching methods. Figure 5 shows an example of what can be done in that area .

When studying movements in a complex gravitational field, my software allows you to launch then a virtual spacecraft and to observe its erratic movement all around the gravity wells. A 3-D representation may induce some visualization of the space curvature...

I am now carrying out such researches. My purpose is to produce such easy to use software so that most teachers may rapidly put computerised methods into practice in their classroom. 